Check for updates

The BMJ

Cite this as: BMJ 2021;372:n612 http://dx.doi.org/10.1136/bmi.n612 Published: 02 March 2021

\title{
Covid-19: Single dose of Pfizer and Oxford vaccines cuts risk of hospital admission by $80 \%$ in over 80 s, data suggest
}

Gareth lacobucci

Both the Pfizer BioNTech and the Oxford AstraZeneca vaccines are highly effective in reducing covid-19 infections and protecting against severe disease in older adults, preliminary data show.

Analysis by Public Health England (PHE), published as a preprint, ${ }^{1}$ estimated that a single dose of either vaccine is around $80 \%$ effective at preventing hospital admission in people aged over 80, three to four weeks after the first dose. A single dose of the Pfizer vaccine also led to an $85 \%$ reduction in deaths from covid-19 in people aged 70 and over, the study suggested. These data are not yet available for the Oxford AstraZeneca vaccine because it was rolled out later.

Mary Ramsay, PHE's head of immunisation, said, "This adds to growing evidence showing that the vaccines are working to reduce infections and save lives. While there remains much more data to follow, this is encouraging, and we are increasingly confident that vaccines are making a real difference."

All adults aged 70 years or older in England (over 7.5 million) were eligible for inclusion in the study, which used a test negative case control design to compare the rate of vaccination in symptomatic people who tested positive for covid-19 with those who tested negative. It also compared hospital admissions and death rates in people in their 8 os who were vaccinated at least two weeks previously with those who were not vaccinated.

Among people aged 70 and over, protection against symptomatic covid-19 after a single dose of the Pfizer vaccine reached $61 \%$ (95\% confidence interval $51 \%$ to $69 \%$ ) from 28 to 34 days after vaccination then plateaued. Protection after a single dose of the Oxford Astra-Zeneca vaccine reached 60\% (95\% CI 41\% to $73 \%$ ) from 28 to 34 days and increased to $73 \%$ (95\% CI $27 \%$ to $90 \%$ ) from day 35 onwards.

Protection after two doses of the Pfizer vaccine increased to around $85-90 \%$, but data are not yet available for the Oxford AstraZeneca vaccine.

People aged 80 and over who had one dose of Pfizer's vaccine and still developed symptoms had an additional 43\% (95\% CI 33\% to 52\%) lower risk of emergency hospital admission and an additional 51\% (95\% CI 37\% to 62\%) lower risk of death. Those vaccinated with one dose of the Oxford AstraZeneca vaccine had an additional $37 \%$ (95\% CI $3 \%$ to $59 \%$ ) lower risk of emergency hospital admission, but there was insufficient follow-up data to assess mortality.

"Combined with the effect against symptomatic disease, this indicates that a single dose of either vaccine is around $80 \%$ effective at preventing hospital admissions and a single dose of the Pfizer vaccine is $85 \%$ effective at preventing death with covid-19," the paper said. The authors added that there was "a clear effect of the vaccines against the UK variant of concern."

Deborah Dunn-Walters, chair of the British Society for Immunology covid-19 and immunology taskforce, said, "These findings are particularly welcome news because the participants were all aged 70 and over. The fact that vaccination is effective in significantly reducing symptomatic cases, hospital admissions, and deaths from covid-19 in this older age group is positive news."

Paul Hunter, professor in medicine at the University of East Anglia, said, "The main conclusions from this paper are that the two vaccines currently in use in the UK appear to be equally effective at preventing infection in older people. Of perhaps even greater importance is that even if people get a symptomatic infection they are still less likely to need to be admitted to hospital and less likely to die."

Lopez Bernal J, Andrews N, Gower C, et al. Early effectiveness of covid-19 vaccination with $\mathrm{BNT} 162 \mathrm{~b} 2 \mathrm{mRNA}$ vaccine and $\mathrm{ChAdO} \mathrm{X} 1$ adenovirus vector vaccine on symptomatic disease, hospitalisations, and mortality in older adults in the UK: a test negative case control study. https://khub.net/documents/135939561/430986542/Early+effectiveness+of+COVID+vaccines.pdf/ffd7161c-b255-8e88-c2dc-88979fc2cc1b?t=1614617945615. 